\title{
Exhaled 8-isoprostane in sarcoidosis: relation to superoxide anion production by bronchoalveolar lavage cells
}

\author{
Wojciech J. Piotrowski • Zofia Kurmanowska • \\ Adam Antczak · Jerzy Marczak • Maciej Ciebiada • \\ Paweł Górski
}

Received: 28 February 2010/Revised: 9 May 2010/Accepted: 11 May 2010/Published online: 3 June 2010

(C) The Author(s) 2010. This article is published with open access at Springerlink.com

\begin{abstract}
Objective This study was designed to examine the mutual relationship between 8-isoprostane in exhaled breath condensate (EBC) and superoxide anion generation by bronchoalveolar lavage fluid (BALF) cells in patients with sarcoidosis.

Design About 29 patients with sarcoidosis, 34 healthy never smokers (control group for EBC) and 15 healthy never smokers (control group for BAL) were examined. EBC was collected directly before bronchoscopy. 8-Isoprostane was measured by ELISA, and superoxide anion by colorimetry.

Results Exhaled breath condensate 8-isoprostane is increased in sarcoidosis (median, 25-75 percentile): 2.50 ; $2.50-3.90$ versus $6.20 ; 2.50-16.95 \mathrm{pg} / \mathrm{ml}, \quad p \leq 0.05$ ). Spontaneous superoxide anion release from BALF cells was significantly elevated only in patients with a high percentage of lymphocytes in BALF $(6.42 \pm 1.24$ vs. $23.52 \pm 4.30 \mathrm{nmol} / 10^{6}$ cells, $\left.p \leq 0.01\right)$. There were no correlations between 8-isoprostane and spontaneous or stimulated superoxide anion release.
\end{abstract}

Responsible Editor: Michael Parnham.

W. J. Piotrowski ( $₫)$ - Z. Kurmanowska · A. Antczak ·

J. Marczak · M. Ciebiada · P. Górski

Department of Pneumology and Allergy,

Medical University of Lodz,

Kopcinskiego Str 22, 90-153 Lodz, Poland

e-mail: piotrow@toya.net.pl

P. Górski

e-mail: p_gorski@toya.net.pl
Conclusions We confirmed higher concentrations of EBC 8-isoprostane in sarcoidosis and higher spontaneous release of superoxide anion from BALF cells in patients with sarcoidosis. The increase of EBC 8-isoprostane is not directly related to superoxide anion released from BALF cells.

Keywords Bronchoalveolar lavage .

Exhaled breath condensate $\cdot$ Oxidative stress $\cdot$ Sarcoidosis . Superoxide anion

\section{Introduction}

Oxidative stress has been implicated in the pathophysiology of many lung diseases. An example is idiopathic pulmonary fibrosis (IPF), a disease characterized by fatal prognosis due to massive lung fibrosis and unresponsiveness to treatment [1]. Increased ex-vivo production of superoxide anion or hydrogen peroxide by cells from bronchoalveolar lavage (BAL) have also been reported in sarcoidosis [2, 3], but secondary markers of oxidative stress, such as oxidized proteins, or oxidized methionine residues in BAL fluid (BALF) are not increased in sarcoidosis, contrary to IPF [4-6].

Exhaled breath condensate is a relatively new, promising and non-invasive method that enables collecting biological material from lower respiratory tract in order to measure various biomarkers [7]. 8-Isoprostane is a marker of oxidative stress. It is a prostaglandin- $\mathrm{F}_{2 \alpha}$ isomer, produced in vivo by free radical-catalyzed peroxidation of arachidonic acid [7-9]. Elevated concentrations of 8-isoprostane in BALF [10] and exhaled breath condensate (EBC) $[11,12]$ in patients with sarcoidosis have been reported. However, the clinical value of this finding is still unknown. There is some hope that this biomarker could be 
helpful in selecting patients with the worst prognosis. Reactive oxygen species (ROS) activate metalloproteinases and inactivate their inhibitors [13, 14], or stimulate cells to release TGF- $\beta$ and other pro-fibrogenic cytokines [15]. Also, alveolar epithelial cells apoptosis can be induced by ROS [16]. All these mechanisms may be involved in the pathogenesis of chronic and progressive lung sarcoidosis.

We have previously reported increased 8-isoprostane in exhaled breath condensate in patients with sarcoidosis [12]. Further, we found that spontaneous, rather than PMA-stimulated, superoxide anion production by BAL cells is increased in sarcoidosis [17]. The present study was planned to evaluate the correlation between both spontaneous and PMA stimulated superoxide anion generation and EBC 8-isoprostane.

\section{Materials and methods}

Study and control groups

Twenty-nine patients with newly diagnosed, histo-pathologically confirmed sarcoidosis were included. All were non-smokers, non-atopic and had never been treated with steroids or immunosuppressive drugs. There were no other co-morbidities. Respiratory infection in the last 4 weeks was an exclusion criterion. Based on the radiological classification, there were 9 patients with stage I, 14 with stage II, and 6 with stage III. The control group for EBC 8-isoprostane consisted of 34 healthy never-smokers, members of the hospital staff and medical students. The control group for superoxide release from BALF cells consisted of 15 healthy non-smokers who had undergone bronchoscopy due to unspecific clinical/radiological signs, finally proved not to be related to any lung pathology. Characteristics of the study and control groups are presented in Table 1.

Bronchoscopy was performed with a flexible bronchoscope (Pentax ${ }^{\circledR}$, Japan) according to British Thoracic
Society Guidelines [18]. Patients optionally received midanium and atropine before the examination, $2 \%$ lidocaine was used as a topical anaesthetic.

Bronchoalveolar lavage fluid (BALF) was collected from medial lobe or lingula by instillation and subsequent withdrawal of $4 \times 50 \mathrm{ml}$ of $0.9 \% \mathrm{NaCl}$. The fluid recovery was $57 \pm 2 \%$. The crude BALF was filtered through gauze, centrifuged, and the pellet was suspended in a phosphate buffer. The total cell count (TCC) was presented as $n \times 10^{6}$. Cytospin slides were prepared and stained by May-Grünwald-Giemsa stain. Numbers of macrophages, lymphocytes, neutrophils and eosinophils were calculated under a light microscope and presented as percent of TCC. Moreover, total cells and particular cell types were presented as number of cells $\left(n \times 10^{4}\right)$ per $\mathrm{ml}$ of recovered fluid.

Superoxide anion $\left(\mathrm{O}_{2}^{-}\right)$production by BALF cells was measured colorimetrically, as previously described [19]. The method is based on the reduction of cytochrome $\mathrm{C}$ by superoxide anion. Superoxide dismutase was used to inhibit the reaction. Spontaneous and excess release after stimulation with PMA ( $1 \mathrm{ng} / \mathrm{ml}$, for $20 \mathrm{~min})$ were measured. Values were expressed as nmol $\mathrm{O}_{2}{ }^{-} / 10^{6}$ cells. Due to the fact that lymphocytes could be neglected as a source of oxidants, we presented these values as nmol $\mathrm{O}_{2}{ }^{-} / 10^{6}$ cells potentially producing superoxide anion (macrophages, neutrophils, eosinophils). This value was calculated according to the following formula: $C=$ $n \times 100 / 100-y$, where $n$ is the production of superoxide by all BALF cells, and $y$ is a percentage of BALF lymphocytes.

\section{Collection of exhaled breath condensate}

The exhaled breath condensate (EBC) was collected using a condensing device (Ecoscreen, Jaeger, Germany). Patients were asked to breathe out spontaneously for 10 min through a mouthpiece equipped with a saliva trap. The respiratory rate ranged from 15 to 20 breaths/min. All

Table 1 Characteristics of the control and study groups

\begin{tabular}{lllllll}
\hline & Control EBC & Control BAL & Sarcoidosis all & Sarcoidosis stage I & Sarcoidosis stage II & Sarcoidosis stage III \\
\hline Gender (females/males) & $20 / 14$ & $7 / 8$ & $13 / 16$ & $5 / 4$ & $6 / 8$ & $2 / 4$ \\
Age (years) & $39.8 \pm 2.6$ & $43.6 \pm 4.0$ & $40.2 \pm 2.0$ & $39.4 \pm 2.8$ & $38.6 \pm 2.8$ & $45.3 \pm 6.0$ \\
FEV $_{1} \%$ predicted & $104.2 \pm 2.7$ & $103.3 \pm 3.1$ & $92.9 \pm 3.0$ & $97.4 \pm 4.1$ & $86.7 \pm 3.4$ & $102.0 \pm 10.1$ \\
FVC \% predicted & $108.6 \pm 3.2$ & $103.0 \pm 3.1$ & $94.8 \pm 3.1$ & $97.6 \pm 5.5$ & $91.9 \pm 3.0$ & $98.0 \pm 11.7$ \\
FEV 1 /FVC \% & $81.2 \pm 1.1$ & $84.4 \pm 1.6$ & $84.9 \pm 1.5$ & $89.0 \pm 2.2$ & $81.5 \pm 1.9$ & $87.2 \pm 3.3$ \\
DLCOc \% predicted & - & - & $83.2 \pm 3.6$ & $91.1 \pm 5.1$ & $79.7 \pm 4.6$ & $73.4 \pm 13.8$ \\
BAL lymphocytes \% & - & $9.5 \pm 1.1$ & $34.7 \pm 4.4$ & $39.3 \pm 8.0$ & $31.6 \pm 5.8$ & $35.0 \pm 12.4$ \\
\hline
\end{tabular}

$B A L$ bronchoalveolar lavage; $D L C O$ diffusion capacity for carbon monoxide corrected for hemoglobin concentration; $E B C$ exhaled breath condensate; $F E V_{l}$ forced expiratory volume in first second of expiration; $F V C$ forced vital capacity 
subjects wore a nose-clip and rinsed their mouths with distilled water just before and in the seventh minute of the condensing process in order to reduce nasal contamination. Samples were stored at $-80^{\circ} \mathrm{C}$ for not longer than 4 weeks until measurements were taken. The collection of EBC was performed following available recommendations [20], always before the bronchoscopy.

8-Isoprostane concentrations in breath condensate were measured by a specific enzyme immonoassay (EIA) kit (Cayman Chemical, Ann Arbor, MI), as previously described [12]. The detection limit was $5 \mathrm{pg} / \mathrm{ml}$. Levels of measured mediators below the detection limit were arbitrarily assumed to be half of the detection limit value. 8-Isoprostane was also measured in BAL, and results were used for estimation of correlations. BAL 8-isoprostane results for the control group are not available.

\section{Statistical analysis}

Data were expressed as mean \pm standard error of means (SEM). The Kolmogorow-Smirnoff test was used to assess normality. Median with 25th and 75th percentiles was provided for non-normally distributed data. Unpaired T-test (for normally distributed data) and Mann-Whitney test (for non-parametric data) were used to compare sarcoidosis with the controls. When more than two groups were compared, one-way ANOVA and Bonferroni post-test (for data with Gaussian distribution) or Kruskall-Wallis followed by Dunn's Multiple Comparison Test (for data without normal distribution) were used. The Spearman test was applied to assess correlations. A $p$ value $\leq 0.05$ was deemed statistically significant.

The study was approved by Ethical Committee at Medical University of Lodz (consent No. RNN/99/08/KE) and all patients signed informed consent.

The funding source had no influence on the study.

\section{Results}

Concentrations of 8-isoprostane in EBC were higher in sarcoidosis (median; $25-75$ percentile: $2.50 ; 2.50-3.90$ vs. $6.20 ; 2.50-16.95 \mathrm{pg} / \mathrm{ml}, p<0.05)$. Although there were no significant differences between radiological stages, the significance was highest when stage III was compared with controls $(26.35 ; 3.90-33.85 \mathrm{pg} / \mathrm{ml}, p<0.01$, Fig. 1) and between controls and patients with low lymphocyte percentage in BALF $(15.10 ; 5.10-34.50 \mathrm{pg} / \mathrm{ml}, p<0.01$, Fig. 2a).

Superoxide anion release, both spontaneous (mean \pm SEM: $6.42 \pm 1.24$ vs. $17.08 \pm 2.87 \mathrm{nmol} / 10^{6}$ cells) and PMA-stimulated excess superoxide release (median; 25-75 percentile: $1.74 ; 0-6.01$ vs. $5.90 ; 0-28.01 \mathrm{nmol} / 10^{6}$ cells),

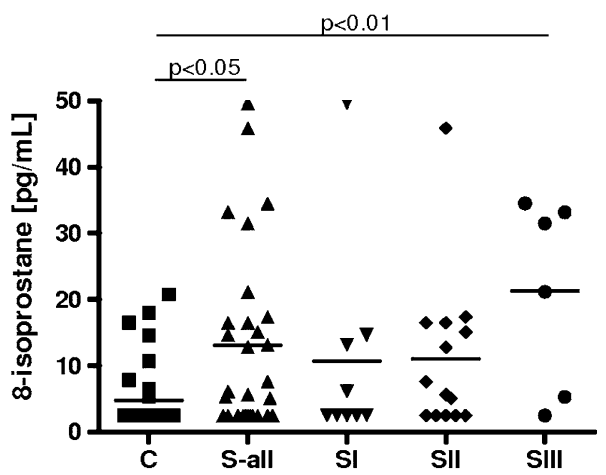

Fig. 1 Comparison of 8-isoprostane concentration in exhaled breath condensate (EBC) of controls (C), all sarcoidosis patients (S) and patients divided according to radiological stages (SI, SII and SIII)
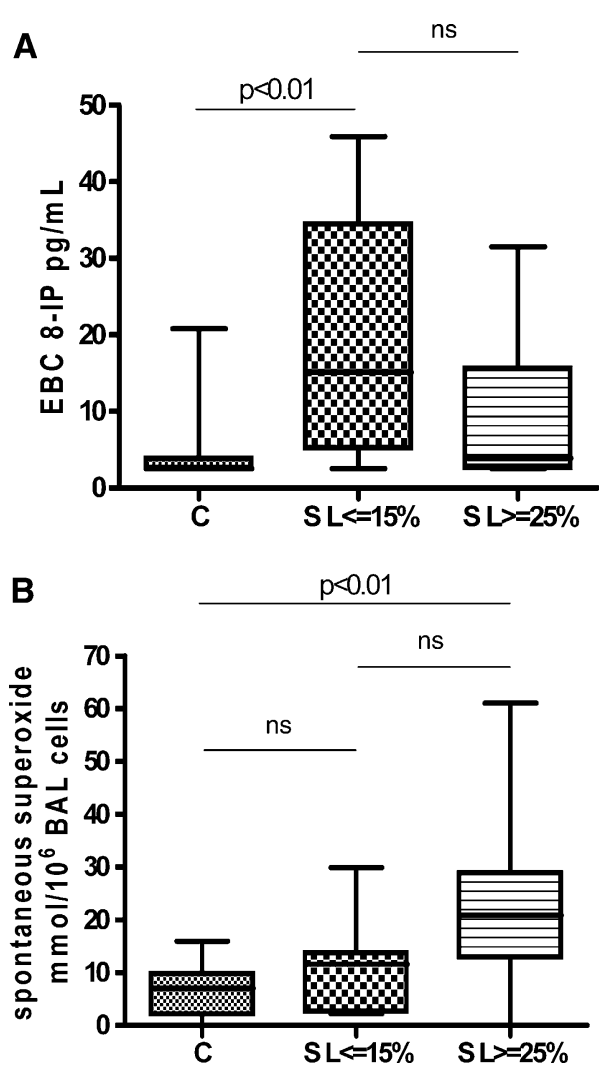

Fig. 2 EBC 8-isoprostane (a) and spontaneous superoxide anion release from BAL cells (b) depending on high and low lymphocyte percentage in BAL fluid versus controls. The bars are limited by 25 th and 75th percentiles, lines within bars represent means, T-bars represent maxima. $C$ controls; $S$ sarcoidosis; $L$ BAL lymphocytes $\leq 15$ or $\geq 25 \%$. Other abbreviations: $B A L$ bronchoalveolar lavage; $E B C$ exhaled breath condensate; $n s$ not significant

in all sarcoidosis patients showed no significant difference compared to controls in this study. Neither in the case of spontaneous nor that of PMA-stimulated release were there any significant differences between patients with different radiological stages. Patients with high lymphocyte 
percentage in BALF had significantly higher concentrations of spontaneous superoxide anion compared to controls (mean \pm SEM: $23.52 \pm 4.30 \mathrm{nmol} / 10^{6}$ cells, $p<0.01$, Fig. 2b).

We did not find any correlations between EBC 8-isoprostane and spontaneous $(r=-0.06 ; p=0.76)$ or PMA-stimulated superoxide anion release $(r=-0.10$; $p=0.61)$. BAL 8-isoprostane was correlated with EBC results $(r=0.64, p=0.0004)$, but was not correlated with spontaneous $(r=0.10, p=0.63)$ or stimulated superoxide release $(r=0.02, p=0.92)$.

Exhaled breath condensate 8-isoprostane concentrations were negatively correlated with the percentage of lymphocytes in BALF ( $r=-0.40 ; p=0.03$ ).

PMA-stimulated superoxide production was positively correlated with the number of neutrophils in BALF ( $r=0.48 ; p=0.009)$.

Bronchoalveolar lavage 8-isoprostane correlated negatively with FVC percent predicted $(r=-0.41, p=0.049)$ and $\mathrm{FEV}_{1} / \mathrm{FVC}$ ratio $(r=-0.49, p=0.02)$.

\section{Discussion}

Although elevated concentrations of 8-isoprostanes in EBC $[11,12]$ and increased release of superoxide anion from alveolar cells after stimulation [2] in patients with sarcoidosis have already been reported, this is the first study on the mutual relationship between EBC 8-isoprostane and both PMA stimulated and spontaneous production of superoxide by BAL cells.

Despite the evident radiological and functional progression, several markers of activity are used in everyday practice, starting from various laboratory markers in serum or BAL, through increased $\mathrm{Ga}^{67}$ uptake in scintigraphy scans, to changes in BALF cellular pattern. None of these markers is specific nor sensitive enough; in addition none adds much to the ability to determine future prognosis and indications to treatment. One of the best markers of activity, elevated percentage of lymphocytes in BALF (with high CD4/CD8 ratio), reflects only the intensity of lymphocytic alveolitis. Highly elevated content of lymphocytes is a typical feature of those with acute onset and evidently better prognosis [21, 22]. Other changes in BALF cellular pattern, however, may have some negative prognostic value. For instance, high content of neutrophils and eosinophils were reported in those with chronic course and increased risk of lung fibrosis [22, 23]. It may be important that these cells are very potent producers of reactive oxygen species (ROS) and other toxic substances, which may be released upon stimulation of NADPH-oxidase, or other ROS-generating intracellular sources. Our finding of higher spontaneous release of superoxide anion from BALF cells of patients with active lymphocytic inflammation suggests that BALF macrophages are constantly stimulated in vivo, and so release superoxide anion spontaneously. Other authors also confirm such a possibility, showing spontaneous release of gamma-interferon from alveolar macrophages and lung $\mathrm{T}$ lymphocytes in sarcoidosis [24]. This type of ROS production seems to be related to the intensity of lymphocytic inflammation, and evidently is not a feature of a more severe disease. In clinical practice, however, the most problematic patients are those with low activity markers, low lymphocyte content in BALF, but with progression of lung changes.

Exhaled breath condensate 8-isoprostane, although increased in sarcoidosis, is not only unrelated to the production of superoxide anion by BALF cells, but seems to show reverse trends in relation to percentage of lymphocytes. The highest levels have been found in patients with radiological stage III. In our previous study [12], we found that increased EBC 8-isoprostane in sarcoid patients was not correlated with BALF lymphocytes, but were correlated with BALF eosinophils, and BALF eosinophils were inversely correlated with DLCO. The present study, dedicated to the mutual relationship between the momentary production of superoxide anion by activated BALF cells and 8-isoprostane in EBC, seems to confirm our earlier observations. These results are also consistent with the results of Montuschi et al. [10], who found that BAL 8-isoprostane in sarcoidosis negatively correlates with BAL lymphocytes.

We provided BAL 8-isoprostane results, but only for the estimation of correlations. We did not find any between BAL 8-isoprostane and superoxide production by BAL cells. We confirmed our earlier findings of a positive correlation between $\mathrm{EBC}$ and $\mathrm{BAL}$ 8-isoprostane [12]. Moreover, weak but negative correlations between $\mathrm{BAL}$ 8 -isoprostane and FVC percent predicted and $\mathrm{FEV}_{1} / \mathrm{FVC}$ ratio confirm our main results.

The lack of BAL 8-isoprostane results for the control group is an important limitation of the present study. The main reason was the lack of consent from the healthy volunteers (EBC control group) to be subjected to bronchoscopy. On the other hand, the control group for BAL, although consisting of healthy subjects, is not an "ideal" control group for EBC. As the primary objective of the study was the estimation of the correlations between exhaled breath 8-isoprostane and superoxide generation, we decided to approve this disadvantage.

One of the limitations of the use of 8-isoprostane in the monitoring of sarcoidosis and other lung diseases is a wide range of results in a study group and a substantial overlapping with control group results. One of the factors that could be responsible for this finding may be different antioxidant potential of individual subjects, which may be 
genetically determined [25]. 8-Isoprostane is not a specific marker for sarcoidosis, therefore other inflammatory diseases may influence the results. Certainly, both the control and study groups were strictly selected towards exclusion criteria, such as infection, atopy and other inflammatory diseases of the respiratory and digestive tracts, but some subclinical states cannot be excluded with $100 \%$ certainty.

The two most likely explanations of the discrepancy between EBC 8-isoprostane and superoxide production by BAL cells are the following:

First, that superoxide anion and other ROS may be scavenged immediately after formation by naturally occurring antioxidants, preventing further peroxidation of membrane phospholipids. Although antioxidant protection was not analyzed in this paper, this possibility should be tested in a separate study.

Second, that other than alveolar inflammatory cells, sources of ROS may play a role in initiation of lipid peroxidation. For instance, alveolar epithelial cells covering a huge area of alveoli are potent sources of reactive oxygen and nitrogen species [26, 27].

The overall conclusion is that 8 -isoprostane and superoxide generation by BAL cells characterize different processes. The latter is related to the intensity of inflammation, and is more common in less severe forms. 8-Isoprostane, related to peroxidation of lipid membranes, may describe chronic destruction linked to more chronic forms and worse prognosis. The latter statement deserves further follow-up studies for confirmation of the prognostic value of EBC 8-isoprostane.

Acknowledgments This study was supported by Medical University of Lodz individual grants: 502-11-451 (Dr W.J. Piotrowski) and 502-11-710 (Prof A. Antczak).

Open Access This article is distributed under the terms of the Creative Commons Attribution Noncommercial License which permits any noncommercial use, distribution, and reproduction in any medium, provided the original author(s) and source are credited.

\section{References}

1. Behr J, Maier K, Degenholb B, Krombach F, Vogelmeier C. Antioxidative and clinical effects of high-dose $\mathrm{N}$-acetylcysteine in fibrosing alveolitis. Adjunctive therapy to maintenance immunosuppression. Am J Respir Crit Care Med. 1997;156:011897.

2. Cassatella MA, Berton G, Agostini C, et al. Generation of superoxide anion by alveolar macrophages in sarcoidosis: evidence for the activation of the oxygen metabolism in patients with high-intensity alveolitis. Immunology. 1989;66:451-8.

3. Fels AOS, Nathan CF, Cohn ZA. Hydrogen peroxide release by alveolar macrophages from sarcoid patients and by alveolar macrophages from normals after exposure to recombinant interferons $\alpha \mathrm{A}, \beta$, and $\gamma$ and 1, 25-Dihydroxyvitamin D3. J Clin Invest. 1987;80:381-6.
4. Lenz A-G, Costabel U, Maier KL. Oxidized BAL fluid proteins in patients with interstitial lung diseases. Eur Respir J. 1996;9: 307-12.

5. Maier K, Leuschel L, Costabel U. Increased levels of oxidized methionine residues in bronchoalveolar lavage fluid proteins from patients with idiopathic pulmonary fibrosis. Am Rev Respir Dis. 1991;143:271-4

6. Lenz AG, Hinze-Heyn H, Schneider A, et al. Influence of inflammatory mechanisms on the redox balance in interstitial lung disease. Respir Med. 2004;98:737-45.

7. Montuschi P. Indirect monitoring of lung inflammation. Net Rev Drug Discov. 2002;1:238-42.

8. Montuschi P. Analysis of exhaled breath condensate in respiratory medicine: methodological aspects and potential clinical applications. Ther Adv Respir Dis. 2007;1:5-23.

9. Montuschi P, Barnes PJ, Roberts LJ. Insights into oxidative stress: the isoprostanes. Curr Med Chem. 2007;14:703-17.

10. Montuschi P, Ciabattoni G, Paredi P, et al. 8-isoprostane as a biomarker of oxidative stress in interstitial lung diseases. Am J Respir Crit Care Med. 1998;158:1524-7.

11. Psathakis K, Papatheodorou G, Plataki M, et al. 8-Isoprostane, a marker of oxidative stress, is increased in the expired breath condensate of patients with pulmonary sarcoidosis. Chest. 2004;125:1005-11.

12. Piotrowski WJ, Antczak A, Marczak J, Nawrocka A, Kurmanowska Z, Górski P. Eicosanoids in exhaled breath condensate and BAL fluid of patients with sarcoidosis. Chest. 2007;132:589-96.

13. Fu X, Kassim SY, Parks WC, Heinecke JW. Hypochlorous acid generated by myeloperoxidase modifies adjacent tryptophan and glycine residues in the catalytic domain of matrix metalloproteinase-7 (matrilysin): an oxidative mechanism for restraining proteolytic activity during inflammation. J Biol Chem. 2003;278:28403-9.

14. Frears ER, Zhang Z, Blake DR, O'Connell JP, Winyard PG. Inactivation of tissue inhibitor of metalloproteinase-1 by peroxynitrite. FEBS Lett. 1996;381:21-4.

15. Bellocq A, Azoulay E, Marullo S, Flahault A, Fouqueray B, Philippe C, Cadranel J, Baud L. Reactive oxygen and nitrogen intermediates increase transforming growth factor-betal release from human epithelial alveolar cells through two different mechanisms. Am J Respir Cell Mol Biol. 1999;21:128-36.

16. Wallach-Dayan SB, Izbicki P, Cohen PY, Gerstl-Golan R, Fine A, Breuer R. Bleomycin initiates apoptosis of lung epithelial cells by ROS but not by Fas/FasL pathway. Am J Physiol Lung Cell Mol Physiol. 2006;290:L790-6.

17. Piotrowski WJ, Kurmanowska Z, Antczak A, Marczak J, Majewski S, Górski P. Superoxide anion production by bronchoalveolar lavage cells in relation to cellular composition and lung function in sarcoidosis and chronic bronchitis. Pol Arch Med Wewn. 2009;119:777-84.

18. British Thoracic Society guidelines on diagnostic flexible bronchoscopy. British Thoracic Society Bronchoscopy Guidelines Committee, a Subcommittee of the Standards of Care Committee of the British Thoracic Society. Thorax 2001; 56(suppl I): i1-i21.

19. Suzuki Y, Lehrer RI. NAD(P)H oxidase activity in human neutrophils stimulated by phorbol myristate acetate. J Clin Invest. 1980;66:1409-18.

20. Horvath I, Hunt J, Barnes PJ, on behalf of the ATS/ERS task force on exhaled breath condensate. Exhaled breath condensate: methodological recommendations and unresolved questions. Eur Respir J. 2005;26:523-48.

21. Winterbauer RH, Lammert J, Selland M, Rae WU, Corley D, Springmeyer SC. Bronchoalveolar lavage cell populations in the diagnosis of sarcoidosis. Chest. 1993;104:352-61. 
22. Drent M, Jacobs JA, de Vries J, Lamers RJS, Liem IH, Wouters EFM. Does the cellular bronchoalveolar lavage fluid profile reflect the severity of sarcoidosis? Eur Respir J. 1999;13: 1338-44.

23. Ziegenhagen MW, Rothe ME, Schlaak M, Müller-Quernheim J. Bronchoalveolar and serological parameters reflecting the severity of sarcoidosis. Eur Respir J. 2003;21:407-13.

24. Robinson BWS, McLemore TL, Crystal RG. Gamma interferon is spontaneously released by alveolar macrophages and lung $\mathrm{T}$ lymphocytes in patients with pulmonary sarcoidosis. J Clin Invest. 1985;75:1488-95.

25. Arcoli JJ, Hokanson JE, Abraham E, Geraci M, Murphy JR, Bowler RP, Dinarello CA, Silveira L, Sanhoff J, Heyland D,
Wischmeyer P, Crapo JD. Extracellular superoxide dismutase haplotypes are associated with acute lung injury and mortality. Am J Respir Crit Care Med. 2008;179:105-12.

26. Piotrowski WJ, Marczak J, Dinsdale D, et al. Release of hydrogen peroxide by rat type II pneumocytes in the prolonged culture. Toxicol In Vitro. 2000;14:85-93.

27. Punjabi CJ, Laskin JD, Pendino KJ, Goller NL, Durham SK, Laskin DL. Production of nitric oxide by type II pneumocytes: increased expression of inducible nitric oxide synthase following inhalation of a pulmonary irritant. Am J Respir Cell Mol Biol. 1994;11:165-72. 